\title{
Reduced breast milk feeding subsequent to cosmetic breast augmentation surgery
}

\section{An absolute \\ rate of one in \\ five women \\ with breast \\ augmentation \\ ... may be \\ unable or \\ unwilling to \\ breast milk}

feed

Christine L Roberts MBBS, FAFPHM, DrPH ${ }^{1.2}$

Amanda J Ampt

PostGradDipClinEp

Charles S Algert $\mathrm{MPH}^{1,2}$

Mark S Sywak MBBS, MMedSc(ClinEpi), FRACS

Jian Sheng C Chen

MStats, MPH, $\mathrm{PhD}^{1,2}$

I Kolling Institute,

Sydney, NSW.

2 University of Sydney,

Sydney, NSW.

christine.roberts@ sydney.edu.au

doi: 10.5694/mjal4.01386
B reastfeeding is beneficial for infants and their mothers. It protects against diarrhoea, respiratory tract and other infant infections, atopic dermatitis, asthma, obesity, diabetes and cancer. ${ }^{1,2}$ Although exclusive breastfeeding achieves optimal infant growth and development, the World Health Organization recognises that providing some breast milk to the infant is better than none. ${ }^{3}$ For mothers, breastfeeding has a contraceptive effect, and reduces the risk of type 2 diabetes, breast cancer and ovarian cancer. $^{1}$

Cosmetic breast augmentation is the most common plastic surgical procedure, and its use is rising dramatically. In Australia, this surgery increased by $150 \%$ between 2001 and 2011. ${ }^{4}$ In the United States, the estimated increase for this period was $45 \%$, although this followed a $550 \%$ increase from 1992 to $2000 .^{5}$ In the United Kingdom, rates increased by $200 \%$ from 2005 to $2013 .{ }^{6}$ In this article, cosmetic breast augmentation (or breast implants) refers to procedures that change the size, shape and texture of healthy breasts. This is distinct from reconstructive breast augmentation, such as following mastectomy.

Although most cosmetic breast surgery occurs among women of reproductive age, there has been little research into pregnancy outcomes, including breastfeeding. A systematic review of breastfeeding outcomes associated with cosmetic breast augmentation surgery identified only three small, observational studies. ${ }^{7}$ One study reported reduced rates of any breastfeeding among women with breast augmentation, while meta-analysis of all three studies suggested a reduced likelihood of exclusive breastfeeding (pooled rate ratio, $0.60 ; 95 \%$ CI, $0.40-0.90) .{ }^{7}$ The authors recommended that studies using larger cohorts and more representative study populations be used to explore the observed association.

Abstract

Objective: To determine the effect of cosmetic breast augmentation on subsequent infant feeding.

Participants, design and setting: Population-based record linkage study of women giving birth in New South Wales, January 2006 - December 2011. Birth records were linked longitudinally to maternal hospitalisations up to 11 years before birth. Breast augmentation was identified by surgical procedure codes in hospital records.

Main outcome measures: Any breast milk feeding at discharge from birth care, and among infants receiving any breast milk, exclusive breast milk feeding. The before-and-after effect of breast augmentation was assessed among women who had the surgery between births.

Results: Among 378389 women who gave birth in the study period, $892(0.2 \%)$ had prior breast augmentation. Among women with breast augmentation, 705 (79\%) provided any breast milk to their infant at discharge, compared with $89 \%$ among women without augmentation. After adjusting for sociodemographic and pregnancy factors, infants of women with breast augmentation were less likely to receive breast milk at discharge than infants of women without augmentation (adjusted relative risk [ARR], 0.90; 95\% Cl, 0.87-0.93). However, infants receiving breast milk were not more or less likely to receive breast milk exclusively (ARR, 0.99; $95 \% \mathrm{Cl}, 0.97-1.01$ ). Women with augmentation surgery between births changed their breastfeeding behaviour (reduced rates), while those with no augmentation or augmentation before both births did not.

Conclusions: Reduced rates of breast milk feeding among women who have undergone breast augmentation underscore the importance of identifying, supporting and encouraging women who are vulnerable to a lower likelihood of breastfeeding.

To test the null hypothesis that augmentation has no effect on breast milk feeding, we conducted a population-based study to determine the effect of cosmetic breast augmentation: (i) on any breast milk feeding in a subsequent pregnancy; and (ii) on exclusive breast milk feeding among women who breast milk fed.

\section{Methods}

The study population was derived from the 391979 women who gave birth in New South Wales from 1 January 2006 to 31 December 2011 (Box 1). As our intention was to examine the effect of cosmetic breast augmentation, women with breast cancer, mastectomy, breast reconstruction or other breast surgery before giving birth were excluded $(n=3831$; Box 1 and Appendix 1). The remaining 388148 women had 506942 births. The first birth in the study period or the first birth after breast augmentation surgery was used in the primary analysis.

Data for the study were obtained from two linked population health datasets: the NSW Perinatal Data Collection (PDC; referred to as birth records) and the NSW Admitted Patient Data Collection (APDC; referred to as hospital records). The PDC is a statutory surveillance system of all births in NSW of at least 20 weeks' gestation or at least $400 \mathrm{~g}$ birthweight. Information on maternal characteristics, pregnancy, labour, delivery, and infant outcomes are recorded by the attending midwife or doctor. The APDC is a census of all NSW inpatient hospital discharges from both public and private hospitals, and day procedure units, and includes demographic and episoderelated data. Diagnoses and procedures are coded for each admission from the medical records according 
1 Study population flowchart, 2006-2011

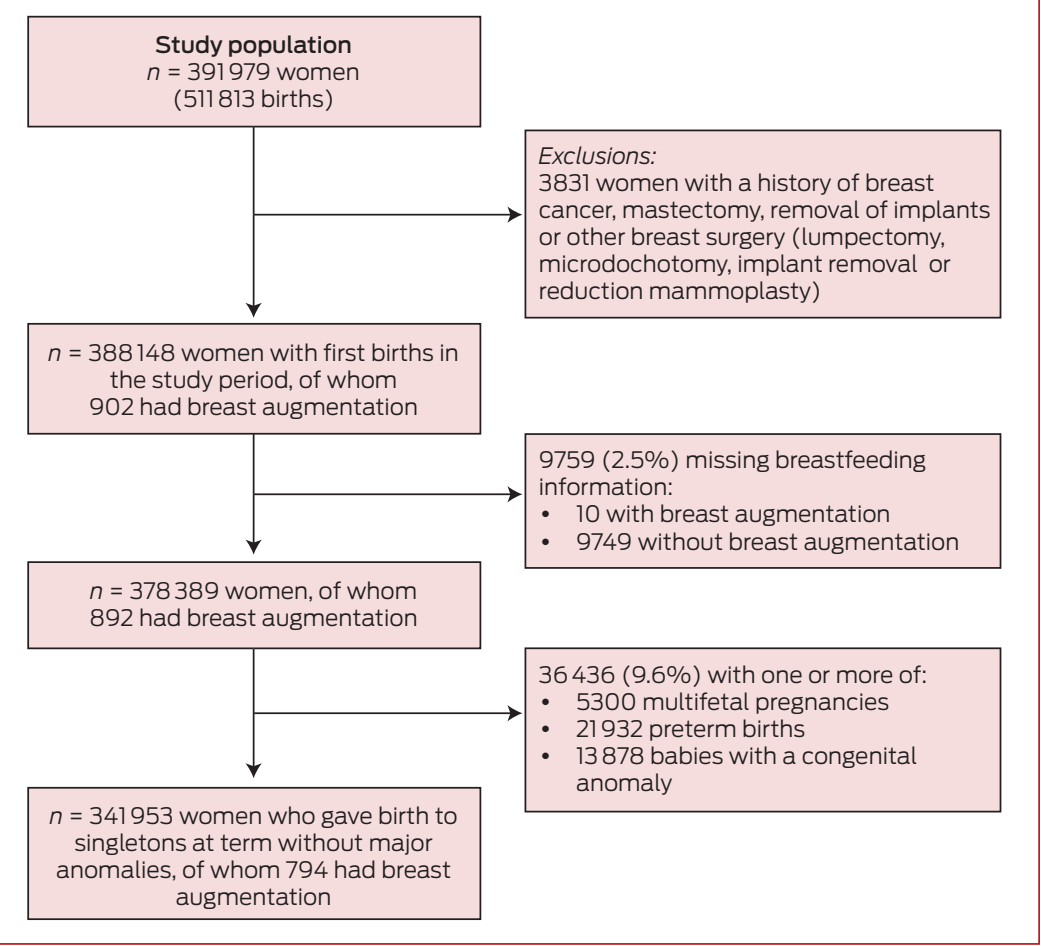

to the International Classification of Diseases, 10th revision, Australian modification (ICD-10-AM) and the Australian Classification of Health Interventions. ${ }^{8}$

Hospital records for individual women were linked cross-sectionally to birth records from 2006 to 2011 and longitudinally (from July 2000 to December 2011). Thus, the minimum lookback period for prior breast surgery ranged from 5.5 to 11.5 years. Record linkage was undertaken by the NSW Centre for Health Record Linkage (CHeReL). For this study, the $\mathrm{CHeReL}$ reported the quality of the record linkage ${ }^{9}$ as 3/1000 false-positive links. We were provided with anonymised data. Ethics approval for the study was obtained from the NSW Population and Health Services Research Ethics Committee.

Breastfeeding information at discharge has been collected in birth records since 2006. One or more of the following three options can be reported in tick-boxes: "breastfeeding", "expressed breast milk" or "infant formula".

The primary outcome was any breast milk feeding (any breast milk, with or without infant formula) at discharge from birth care. Consistent with other studies, ${ }^{7}$ the secondary outcome was exclusive breast milk feeding (only breast milk, either directly from the breast and/or as expressed breast milk) among those with any breast milk feeding.

The exposure of interest was cosmetic breast augmentation, which has a specific surgical procedure code (4552800) in the Australian Classification of Health Interventions. ${ }^{4,8}$ This code is distinct from unilateral breast augmentation and breast augmentation following mastectomy. Hospital records from 2000 onwards were available for identification and date of surgery.

Other factors potentially predictive of breast milk feeding at discharge from maternity care that were available for analysis included: maternal age, country of birth, socioeconomic status according to the Australian Bureau of Statistics Index of Relative Socio-economic Disadvantage, ${ }^{10}$ marital status, urban or rural residence, private care, parity, multifetal pregnancy, antenatal care before 20 weeks' gestation, smoking during pregnancy, morbid obesity, hypertensive disorders of pregnancy, diabetes (pregestational or gestational), labour analgesia, labour induction or augmentation, mode of birth, severe maternal morbidity, ${ }_{1}^{11}$ maternal postnatal length of stay, gestation, small for gestational age $(<10$ th birthweight for gestational age percentile), major congenital anomalies (eg, cleft lip or palate, spina bifida, tracheoesophageal fistula), neonatal intensive care unit admission, and perinatal mortality. These factors are known to be reliably reported. ${ }^{12}$

\section{Statistical analysis}

Descriptive statistics were used to summarise the distributions of maternal and pregnancy characteristics among all women with and without breast augmentation. Poisson regression modelling with robust standard errors ${ }^{13}$ was employed to determine the association of breast augmentation with (i) any breast milk feeding (compared with none) and (ii) exclusive breast milk feeding (compared with non-exclusive) among the "any breast milk feeding" group.

To avoid confounding by factors likely to be associated with reduced breastfeeding, ${ }^{14,15}$ regression analyses were limited to women who had a singleton infant with no major congenital anomalies and born at term ( $\geqslant 37$ weeks). Crude and adjusted relative risks (RRs) with 95\% confidence intervals were estimated for characteristics likely to be associated with breastfeeding.

Finally, among women with at least two births in the study period, we examined the primary and secondary breastfeeding outcomes across births in the following groups: no breast augmentation, breast augmentation between births, and breast augmentation before both births. The before-and-after effect of breast augmentation among women who had breast augmentation surgery between births was assessed using the McNemar test of paired data, with continuity correction.

\section{Results}

Of the 388148 women who were eligible for the study, 902 had documentation of cosmetic breast augmentation surgery (Box 1). Breastfeeding 
2 Maternal, pregnancy and birth characteristics for participants, by breast augmentation status

Mother's age at birth (missing = 106
$<20$ years
20 to $<35$ years
$\geqslant 35$ years
Region of birth (missing $=1489$ )
Australia or New Zealand
Asia
Other

Married or de facto

Socioeconomic status ( missing $=6140$ )

Most disadvantaged

Disadvantaged

Average

Advantaged

Most advantaged

Urban residence at birth

Private care

Nulliparous

Multifetal pregnancy

First antenatal visit $<20$ weeks' gestation

Smoking during pregnancy

Hypertensive disorders

Diabetes

Morbid obesity

Regional labour analgesia

Labour induction

Mode of birth ( missing $=287$ )

Unassisted vaginal

Instrumental vaginal

Caesarean section

Severe maternal morbidity

Mother's postnatal length of hospital stay

$$
\begin{aligned}
& 1-2 \text { days } \\
& 3-4 \text { days } \\
& 5-6 \text { days } \\
& \geqslant 7 \text { days }
\end{aligned}
$$

Preterm birth (<37 weeks' gestation)

Small for gestational age

Neonatal intensive care unit admission

Major congenital anomalies

Perinatal mortality

Infant feeding at discharge

Any breast milk feeding

No breast milk feeding (formula only)

Exclusive breast milk feeding among women who provided any breast milk

Breast-related readmission within 6 weeks
Breast augmentation No breast augmentation

$(n=892)$, no. $(\%) \quad(n=377497)$, no. $(\%)$

$3(0.3 \%)$
$608(68.2 \%)$
$281(31.5 \%)$
$761(85.5 \%)$
$45(5.1 \%)$
$84(9.4 \%)$
$718(80.5 \%)$
$103(11.6 \%)$
$134(15.1 \%)$
$159(17.9 \%)$
$210(23.7 \%)$
$282(31.8 \%)$
$653(73.2 \%)$
$370(41.5 \%)$
$378(42.4 \%)$
$18(2.0 \%)$
$834(93.5 \%)$
$85(9.5 \%)$
$70(7.9 \%)$
$32(3.6 \%)$
$084(31.8 \%)$
$256(28.7 \%)$
$485(54.4 \%)$
$130(14.6 \%)$
$276(31.0 \%)$
$12(1.4 \%)$

$327(37.2 \%)$

$359(40.8 \%)$

$168(19.1 \%)$

$25(2.8 \%)$

$61(6.8 \%)$

75 (8.4\%)

$119(13.3 \%)$

$36(4.0 \%)$

0

705 (79.0\%)

$187(21.0 \%)$

$653(92.6 \%)$

$13(1.4 \%)$

$103368(27.4 \%)$

$4471(1.2 \%)$

$$
\begin{gathered}
15406(4.1 \%) \\
276043(73.2 \%) \\
85942(22.8 \%)
\end{gathered}
$$

$<0.001$

$264041(70.2 \%)$

$58811(15.6 \%)$

$53158(14.1 \%)$

308709 (81.8\%)

0.32

$<0.001$

79232 (21.3\%)

$71517(19.3 \%)$

75027 (20.2\%)

71656 (19.3\%)

73929 (19.9\%)

$263218(69.7 \%)$

0.02

$120211(31.8 \%)$

$<0.001$

206078 (54.6\%)

$5282(1.4 \%)$

344892 (91.4\%)

45073 (11.9\%)

38568 (10.2\%)

$26621(7.1 \%)$

$1277(0.3 \%)$

$101925(27.0 \%)$

210506 (55.8\%)

$51447(13.6 \%)$

115258 (30.6\%)

$$
6102 \text { (1.6\%) }
$$

132944 (35.7\%)

157913 (42.4\%)

$70634(19.0 \%)$

10869 (2.9\%)

21871 (5.8\%)

0.18

35722 (9.5\%)

0.28

$53510(14.2 \%)$

0.48

$13842(3.6 \%)$

8

0.50

0.89

$\begin{array}{cc}334250(88.5 \%) & <0.001 \\ 43247(11.5 \%) & \\ 308552(92.3 \%) & 0.76\end{array}$

0.42

$* \chi^{2}$ test.

information at discharge was missing in 9759 records $(2.51 \%)$. Among the remaining 378389 women, 892 ( $0.24 \%)$ had breast augmentation before a birth. The median age at the time of breast augmentation surgery was 28 years (range, $18-43$ years), and the median interval between surgery and birth was 3.1 years (range, 1.0-10.1 years).

Maternal, pregnancy and birth characteristics for all women with and without breast augmentation are presented in Box 2. At discharge, 705 women $(79.0 \%)$ with breast augmentation provided any breast milk to their infants, compared with $88.5 \%$ of women without breast augmentation.

Breast milk feeding outcomes were then assessed among 341953 singleton infants with no major congenital anomalies born at term. Compared with women without, women with breast augmentation had reduced likelihood (adjusted RR, 0.90; 95\% CI, 0.87-0.93) of feeding their infant with any breast milk at the time of discharge from birth care. Factors controlled for that were positively associated with breast milk feeding included: older maternal age, nonAustralian-born, high socioeconomic status, nulliparity, non-smoker, no obstetric interventions, and longer hospitalisation after birth (Appendix 2). Women with breast augmentation in the 2 years preceding birth had similar rates of any breast milk feeding to women with a longer period since breast surgery $(77 \%$ v $81 \%$; $P=0.17$ ).

For women whose infants received any breast milk, there was no association between breast augmentation and exclusive breast milk feeding. Among these, 593 women (94.0\%) with breast augmentation exclusively breast milk fed. The adjusted RR for exclusive breast milk feeding among women with breast augmentation, compared to those without, was 0.99 (95\% CI, 0.97-1.01).

Among the 106835 women with two births during the study period, 106593 had no record of breast augmentation, 167 had breast augmentation before both births, and 75 had breast augmentation between the two births. The rates of any breast 
milk feeding and exclusive breast milk feeding at the first and second births were compared for these three groups of women (Box 3). The rate of any breast milk feeding was the same for both births among women with no augmentation (87\%). Among women with breast augmentation between the births, the rate declined from $87 \%$ in the first birth to $72 \%$ in the second birth $(P=0.02)$. There was no evidence of significant change among women with augmentation before both births $(77.2 \%$ v $73.7 \% ; P=0.29$; Box 3, A). However, among women who provided any breast milk, the rate of exclusive breast milk feeding was similar in first and second births for women with and without breast augmentation (Box 3, B).

\section{Discussion}

This is the first study to document the population prevalence of cosmetic breast augmentation in a maternity population, and the largest to compare breast milk feeding outcomes for women with and without cosmetic breast augmentation. We found that women with breast augmentation are less likely to provide their infants with any breast milk at the time of discharge. However, among women who provide breast milk, women with breast augmentation are no more or less likely to exclusively breast milk feed their infants. Both the main population analysis and the subgroup analysis of women with breast augmentation between births showed lower rates of any breast milk feeding following augmentation surgery. This consistency of findings strengthens the case that there is an effect, although possible mechanisms are unclear.

Uptake of breast augmentation surgery is increasing, with 8000 Australian, 10000 British and 307000 American women undergoing the procedure in 2011. -6 $^{-6}$ We found that $79 \%$ of these women can be expected to breast milk feed at discharge, compared with $89 \%$ of women without surgery. As maternity care affects breastfeeding success, ${ }^{2}$ these findings underscore the importance of identifying, supporting and encouraging all women who are vulnerable to a lower likelihood of breastfeeding.

Underlying breast hypoplasia and insufficient lactogenesis have been suggested as a reason for reduced breastfeeding rates among women with breast augmentation. ${ }^{16}$ However, we found that among women who had breast augmentation between births, any breast milk feeding fell from $87 \%$ in the "before augmentation" birth to $72 \%$ in the "after augmentation" birth, while the rates in comparison groups remained stable. A demonstrated ability to provide breast milk before augmentation surgery suggests that hypoplasia is not the explanation for lower breastfeeding rates among women with breast augmentation. Similar to the one existing population-based study, ${ }_{1}^{17}$ we found no association between breast augmentation and adverse birth outcomes, including preterm birth, small for gestational age, congenital anomalies, neonatal intensive care unit admission or perinatal death.

Lower breastfeeding rates may reflect maternal and family attitudes and expectations, may be a consequence of surgery, or the breast implants may reduce the ability to lactate. Although a variety of health outcomes have been investigated among women who have silicone breast implants, and their breast milk fed infants, epidemiological studies have not substantiated links with adverse outcomes..$^{18-21}$ Nevertheless, women with breast implants may fear transmitting silicone or other breast implant materials into breast milk. They may also fear, or have been told by their surgeon, that breastfeeding could undo a satisfactory augmentation result. Another explanation is that lactiferous ducts, glandular tissue or nerves of the breast are damaged during surgery, or by pressure from the implants on breast tissue. ${ }^{22}$ Furthermore, complications of the surgery including capsular contracture, haematoma formation, infection or pain may reduce the ability or desire to breastfeed. ${ }^{22}$ Future qualitative research is needed to better understand why women with prior breast augmentation are less likely to breastfeed.
3 Breast milk feeding outcomes for women with two births, showing the before-and-after effect of breast augmentation, 2006-2011

A: Any breast milk feeding

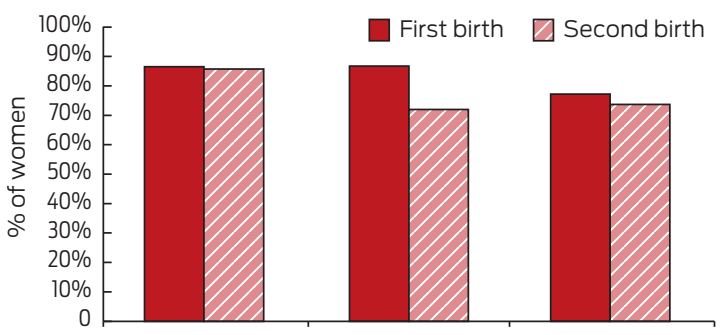

B: Exclusive breast milk feeding

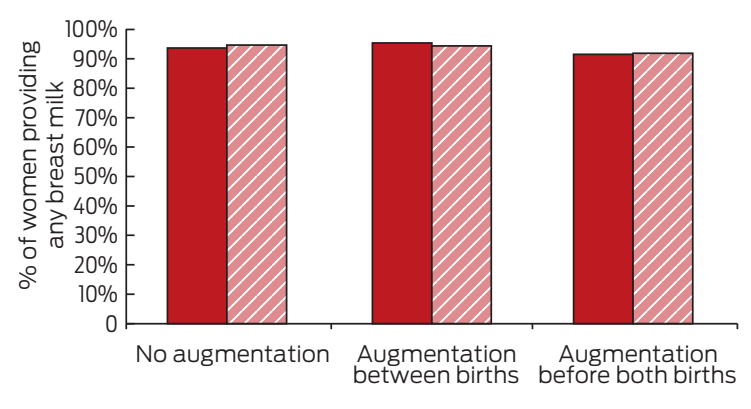

Our findings of reduced rates of any breastfeeding are consistent with the only study that reported rates of any breastfeeding after augmentation among women who attempted breastfeeding. ${ }^{16}$ However, the latter study reported a stronger effect at 2 weeks postpartum (RR, $0.67 ; 95 \% \mathrm{CI}$, $0.50-0.91)$. In contrast, our findings differ from the systematic review of three small studies, which found women with breast implants who breast milk fed were less likely to exclusively breastfeed. ${ }^{7}$ We believe our whole-population findings are more robust. The previous studies had selected populations (eg, lactation referral clients) and variable end points (eg, exclusive breastfeeding, insufficient lactogenesis), used historical controls and made limited attempts to control for potential confounders. ${ }^{7}$ However, it is possible that differences in the rates of exclusive breastfeeding may become apparent after discharge, as follow-up in the three studies was longer (minimum 2 weeks postpartum).

A strength of our study is the use of recent, large, linked population health datasets that include a third of all births in Australia. Breastfeeding information is reported by a midwife, and previous validation studies show 
events occurring around birth or immediately postpartum are well reported. ${ }^{12}$ Longitudinal record linkage allowed the ascertainment of cosmetic breast augmentation surgery. Although a longer lookback period may have increased case ascertainment, ${ }^{23}$ some missed cases among a population of more than 300000 women without breast augmentation are unlikely to change the findings. Similarly, women who have cosmetic surgery overseas or interstate are not captured in this study. Identification of breast augmentation surgery in routinely collected data has not been evaluated but, in general, surgical procedures are reliably identified in hospital discharge data, and other breast surgery, such as mastectomy, is accurately reported (sensitivity, 97\%; positive predictive value, $97 \%) .{ }^{12,24}$

Another strength is that breastfeeding was assessed at the same time for both exposed and unexposed women, unlike prior studies. ${ }^{7}$ The $89 \%$ breastfeeding rate at discharge in our study is similar to the rate reported in the Australian National Infant Feeding Survey $(90.2 \%$ for $<1$ month). ${ }^{25}$

However, information on breastfeeding initiation was not available. If women with breast augmentation initiated breastfeeding but gave up before discharge, the rate of exclusive breastfeeding could be lower if these women were included in the "any breastfeeding" denominator. Another limitation of the study is that breastfeeding is only assessed at one time point (discharge). Breastfeeding rates decline steadily over the first months of infancy ${ }^{25}$ and it is unclear whether this decay would be the same for women with and without breast augmentation. Information was not available on intention to breast milk feed, paternal support for breastfeeding, nor on the details of the breast augmentation surgery, such as the incision type or the type and volume of the breast implant.

An absolute rate of one in five women with breast augmentation who subsequently give birth may be unable or unwilling to breast milk feed their infants. This information should be provided as part of informed decision making to women contemplating breast augmentation surgery.

Acknowledgements: This work was supported by an Australian National Health and Medical Research Council (NHMRC) Centre for Research Excellence Grant (1001066). Christine Roberts is supported by an NHMRC Senior Research Fellowship (APP1021025) and Amanda Ampt is supported by the Dr Albert S McKern Research Scholarship. We thank the NSW Ministry of Health for access to the population health data and the CHeReL for linking the datasets. Jian Sheng (Charles) Chen died just after the completion of this project, and we intend this work to be a tribute and a further example of the extraordinary contribution his work has made to the analysis of linked health data.

Competing interests: No relevant disclosures.

Received 29 Sep 2014, accepted 10 Dec 2014 .

References are available online at www.mja.com.au. 
1 Ip S, Chung M, Raman G, et al. Breastfeeding and maternal and infant health outcomes in developed countries. Evid Rep Technol Assess (Full Rep) 2007; 153: 1-186.

2 Stuebe AM, Schwarz EB. The risks and benefits of infant feeding practices for women and their children. J Perinatol 2010; 30: 155-162.

3 World Health Organization. WHO Global Data Bank on Infant and Young Child Feeding. http://www.who.int/ nutrition/databases/infantfeeding/en (accessed May 2014).

4 Australian Institute of Health and Welfare. Procedures cubes for 2000-01 to 2011-12. Canberra: AlHW, 2013. http://www.aihw.gov.au/ hospitals-data/procedures-data-cubes (accessed May 2014).

5 American Society of Plastic Surgeons. 2012 plastic surgery procedural statistics. http://www. plasticsurgery.org/news/plasticsurgery-statistics/2012-plastic-surgerystatistics.html (accessed Sep 2014).

6 British Association of Aesthetic Plastic Surgeons. Annual Audit. http://baaps. org.uk/about-us/audit (accessed Nov 2014).

7 Schiff M, Algert CS, Ampt A, et al. The impact of cosmetic breast implants on breastfeeding: a systematic review and meta-analysis. Int Breastfeed J 2014; 9: 17. http://www. internationalbreastfeedingjournal. com/content/9/1/17 (accessed Dec 2014).

8 National Centre for Classification in Health. The international statistical classification of disease and health related problems, 10th revision and the Australian modification (ICD-10AM), Australian classification of health interventions, 7th ed. Sydney: NCCH, 2010.
9 Centre for Health Record Linkage. Quality assurance. http://www.cherel. org.au/quality-assurance (accessed Nov 2014).

10 Australian Bureau of Statistics. Information paper: an introduction to Socio-Economic Indexes for Areas (SEIFA). Canberra: ABS, 2006. (ABS Cat. No. 2039.0.) http://www.abs.gov. au/ausstats/abs@.nst/mf/2039.0 (accessed Apr 2014).

11 Roberts CL, Cameron CA, Bell JC, et al. Measuring maternal morbidity in routinely collected health data: development and validation of a maternal morbidity outcome indicator. Med Care 2008; 46: 786-794.

12 Lain SJ, Hadfield RM, Raynes-Greenow $\mathrm{CH}$, et al. Quality of data in perinatal population health databases: $a$ systematic review. Med Care 2012; 50 : e7-e20.

13 Zou G. A modified Poisson regression approach to prospective studies with binary data. Am J Epidemiol 2004; 159: 702-706.

14 Donath SM, Amir LH. Effect of gestation on initiation and duration of breastfeeding. Arch Dis Child Fetal Neonatal Ed 2008; 93: F448-F450.

15 Taylor LK, Lim K, Neville SE. Newborn feeding practices at the time of discharge from hospital in NSW in 2007: a descriptive study. NS W Public Health Bull 2009; 20: 177-181.

16 Cruz NI, Korchin L. Breastfeeding after augmentation mammaplasty with saline implants. Ann Plast Surg 2010; 64: 530-533.

17 Hemminki E, Hovi SL, Sevón T, AskoSeljavaara S. Births and perinatal health of infants among women who have had silicone breast implantation in Finland, 1967-2000. Acta Obstet Gynecol Scand 2004; 83: 1135-1140.
18 Deapen DM, Brody GS. Cancer risk among cosmetic breast implant patients: an update of the Los Angeles study. Plast Reconstr Surg 2012; 129 : 575e-576e.

19 Kjøller K, Friis S, Lipworth L, et al. Adverse health outcomes in offspring of mothers with cosmetic breast implants: a review. Plast Reconstr Surg 2007; 120 (7 Suppl 1): 129S-134S.

20 Kjøller K, Friis S, Mellemkjaer L, et al. Connective tissue disease and other rheumatic conditions following cosmetic breast implantation in Denmark. Arch Intern Med 2001; 16l: 973-979.

21 Winther JF, Friis S, Bach FW, et al. Neurological disease among women with silicone breast implants in Denmark. Acta Neurol Scand 2001; 103: 93-96.

22 Michalopoulos K. The effects of breast augmentation surgery on future ability to lactate. Breast J 2007; 13: 62-67.

23 Chen JS, Roberts CL, Simpson JM, Ford JB. Use of hospitalisation history (lookback) to determine prevalence of chronic diseases: impact on modelling of risk factors for haemorrhage in pregnancy. BMC Med Res Methodol 2011; $11: 68$.

24 Henderson T, Shepheard J, Sundararajan V. Quality of diagnosis and procedure coding in ICD-10 administrative data. Med Care 2006; 44: 1011-1019.

25 Australian Insitute of Health and Welfare. 2010 Australian national infant feeding survey: indicator results. Canberra: AlHW, 2011. (AlHW Cat. No. PHE 156.) http://www.aihw.gov.au/ publication-detail/?id=10737420927 (accessed May 2014) 